\title{
ADHESION FORCE MEASUREMENTS ON SINGLE GECKO SETAE
}

\author{
Yiching A. Liang1, Kellar Autumn², S. Tonia Hsieh', Wolfgang Zesch4, \\ Wai Pang Chan ${ }^{5}$, Ronald S. Fearing ${ }^{6}$, Robert J. Full, Thomas W. Kenny ${ }^{1}$
}

'Department of Mechanical Engineering, Stanford University, Stanford, CA 94305

${ }^{2}$ Department of Biology, Lewis \& Clark College, Portland, OR 97129

${ }^{3}$ Department of Organismic and Evolutionary Biology, Harvard University, Cambridge, MA 02138

${ }^{4}$ Department of MTS, Zuehlke Engineering AG, CH-8952 Schlieren-Zurich

${ }^{5}$ Department of Integrative Biology, University of California at Berkeley, Berkeley, CA 94720

${ }^{6}$ Department of Electrical Engineering and Computer Science, U. of California at Berkeley, Berkeley, CA 94720

\begin{abstract}
Geckos are known for their exceptional ability to climb up vertical walls. The adhesion mechanism has been the subject of debate for nearly a century. It is commonly assumed in the biology community that this remarkable wall-climbing ability in geckos is attributed to van der Waals forces [1]. In this theory, the tiny hairlike structures on the bottom of gecko feet, known as setae, form van der Waals bonds with the surface to facilitate adhesion. These forces are on the order of $\mu \mathrm{N}$ per seta. Various attempts have been made to experimentally quantify the adhesive forces on whole animals, but none had been able to measure the forces on single sctac. Recent development in MEMS tcchnology has produced numerous highly sensitive devices for small force measurements, including a piezoresistive cantilever capable of simultaneous force detections in two directions [2]. We have successfully performed series of adhesion measurements on single setae using this device to support the van der Waals adhesion hypothesis.
\end{abstract}

This paper describes the setup and procedures used to obtain adhesive force measurements on single gecko setae. Experimental results showed that adhesion increases with increasing contact preload and lateral pulling speed. Our data also revealed some interesting characteristics of setal adhesion. The magnitude of the adhesive forces measured supports the hypothesis that individual setae operate by van der Waals forces and sum to generate sufficient forces to explain macroscopic behavior such as clinging or vertical running.

\section{INTRODUCTION}

Tokay geckos (Gekko gecko) are one of the largest Gekkonid lizards (snout-vent length of approximately $37 \mathrm{~cm}$ ) and are found primarily in Southeast Asia. They have large toe pads and are remarkable in their ability to climb up vertical surfaces. Studies on whole animals have found that they are capable of sustaining over 40 times their own body weights on a nearly vertical $\left(85^{\circ}\right.$ to the horizontal) acetate surface [3].

Figure 1 shows the morphology of a gecko foot. The toe pads are covered with leaf-like arrays termed lamellae. The ventral surfaces of the lamellae are densely packed with arrays of keratinous hairs called setae. On average, a gecko has approximately one million setae, each $30-130 \mu \mathrm{m}$ in length and approximately $5 \mu \mathrm{m}$ in diameter. The shorter setae are generally proximal and the longer ones distal. Each seta divides into branches before terminating in a hundred to a thousand projection tips called spatulae. Spatulae are approximately $0.2 \mu \mathrm{m}$ in diameter $[4,5]$.
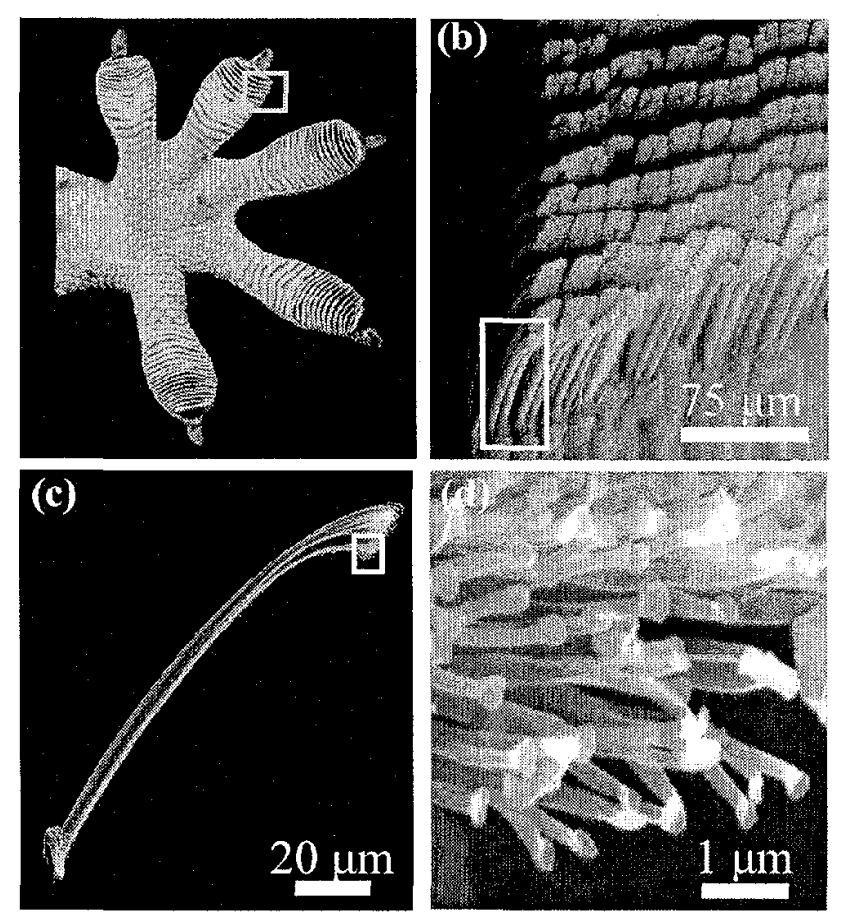

Figure 1. SEM images of (a) the foot of a Tokay gecko (photo courtesy of Mark Moffett); (b) an array of lamellae; (c) an isolated Seta; (d) spatula-shaped tips [1].

Although much attention has been given to the structure of setae, the mechanism of setal adhesion is still controversial, and has been debated for nearly a century. Table 1 lists the various theories suggested. All but the last one has been rejected based on experimental results.

Table 1. Theories suggested for the adhesion mechanism.

\begin{tabular}{|l|l|}
\hline Theory & Reasons for rejection \\
\hline Friction [6] & Adhesion is much higher than friction [1]. \\
\hline Suction [7,8] & Setae can adhere in vacuum [9]. \\
\hline $\begin{array}{l}\text { Capillary wet } \\
\text { adhesion }\end{array}$ & $\begin{array}{l}\text { Skin glands are not present on gecko fect [6, } \\
\mathbf{9 , 1 0 ]}\end{array}$ \\
\hline $\begin{array}{l}\text { Micro- } \\
\text { interlocking [6] }\end{array}$ & $\begin{array}{l}\text { Surface irregularities on the scale of the } \\
\text { spatulae not necessary for adhesion [11]. }\end{array}$ \\
\hline $\begin{array}{l}\text { Electrostatics } \\
{[\mathbf{1 2}]}\end{array}$ & $\begin{array}{l}\text { X-ray bombardment does not affect } \\
\text { adhesion; setae can function in ionized air [9] }\end{array}$ \\
\hline $\begin{array}{l}\text { van der Waals } \\
\text { forces [5, 13] }\end{array}$ & None so far.) \\
\hline
\end{tabular}


The adhesion by van der Waals forces hypothesis is supported by an observation that adhesive force of a whole gecko increases with increasing surface energy of the substrate [13].

Irschick [3] performed experiments to measure the macroscale wall climbing abilities of whole animals. A live gecko with $227.1 \mathrm{~mm}^{2}$ of pad area could sustain an average clinging force of 43.4 N. Assuming the force is divided evenly among all setae, calculated using the typical setal density of $5300 \mathrm{setae} / \mathrm{mm}^{2}[\mathbf{5}]$, this is equivalent to approximately $17 \mu \mathrm{N}$ of force per seta. Although in reality this value can be several times higher since it is unlikcly that all setac arc used at the same time. Experiments of this sort provide rough measurements of the magnitude of wholeanimal adhesion. They are however not sufficient for concluding what mechanism is actually responsible for the adhesion.

Theoretical calculations of the complete van der Waals force between two complex macro-scale objects are simply too complicated to perform [14], and therefore adhesion force measurements on smaller sub-units, such as individual setae, are necessary for verifying the van der Waals mechanism.

\section{THEORY}

van der Waals forces are attractive forces between two nonpolar objects. They are caused by fluctuations in the instantaneous dipole moments of these two objects due to the uneven distribution of electrons in their electron clouds. Two adjacent particles tend to synchronize their dipole moment fluctuations to minimize the total potential energy; therefore van der Waals forces are usually attractive in nature. van der Waals forces are the smallest among all intermolecular forces, but they become significant when a large number of particles are involved. We can calculate the van der Waals attraction between two solids by integrating the London dispersion energy over all particles in both volumes, and differentiating with respect to the separation distance between them [15].

A single spatula adhering to a surface can be roughly modeled as the interaction between two parallel surfaces.

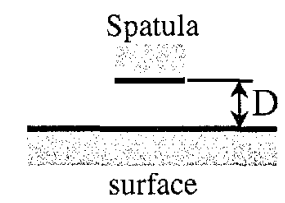

Figure 2. Spatula and surface approximated as two parallel surfaces in contact.

van der Waals force for the configuration in Figure 2 is

$$
F_{v d W}=\frac{A}{6 \pi D^{3}} \quad \text { per unit contact area, }
$$

where $A$ is the Hamaker constant that depends on the materials of the two surfaces. This constant is typically on the order of $10^{-19} \mathrm{~J}$ between two solids, and does not vary significantly for different materials. A separation distance of $D=3 \AA$ is typically used for two solids in real contact. Since $F_{v a w}$ is inversely proportional to the third power of $D$, the attractive force is negligible when the separation distance is more than tens of Angstroms. Although the exact Hamaker constant between the sensor and setae is unknown, the nominal value of $10^{-19} \mathrm{~J}$ can be used to obtain a reasonable approximation. The force is calculated to be approximately 200 $\mu \mathrm{N} / \mu \mathrm{m}^{2}$. This corresponds to $200 \mathrm{~N}$ of attractive force for $1 \mathrm{~mm}^{2}$ of real contact area.

Note that there is a significant difference between real and apparent contact areas. Solid surfaces are rarely ideally planar. Therefore the real contact area is merely the total of the areas between the few opposing asperities actually in position to touch each other [16].

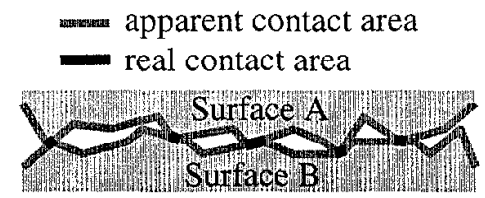

Figure 3. Real vs. apparent contact areas between two surfaces.

Since only the real contact area is considered in the calculation, van der Waals forces between most solid surfaces are negligible. But if one of the surfaces is sufficiently compliant to allow deformation under modest loads, the real contact area can be substantial. A gecko seta in contact with a hard surface is a good cxamplc of this kind of interface. The compliant setal structure can conform to the topography of a surface to form large area of very intimate contact, which increases the magnitude of van der Waals forces.

Since the tips of the spatulae are cup-shaped, only the perimeters are in real contact with the surface. For a seta with one thousand spatulae at $\phi 0.2 \mu \mathrm{m}$, a total van der Waals force of over $200 \mu \mathrm{N}$ results even if only $2.5 \%$ of the total tip area is in real contact with the surface. Note that this value is a very rough estimate. The actual contact area is difficult to quantify due to the irregular shapes and unknown numbers of the spatulae on each seta. It varies greatly between setae and depends also on the roughness of the surface. More accurate values can be calculated with better shape and size estimates on the specific seta sample. Clearly, larger setae would be able to provide larger sticking forces due to the larger contact area. The specific Hamaker constant that corresponds to the silicon-keratin interface can also be used to obtain more accurate force calculations.

\section{DEVICE}

All adhesive force measurements in this paper are obtained using a micromachined dual-axis piezoresistive force sensor [2] shown here with a seta in Figure 4. The device is fabricated on SOI (Silicon-On-Insulator) wafers formed using the SIMOX process.
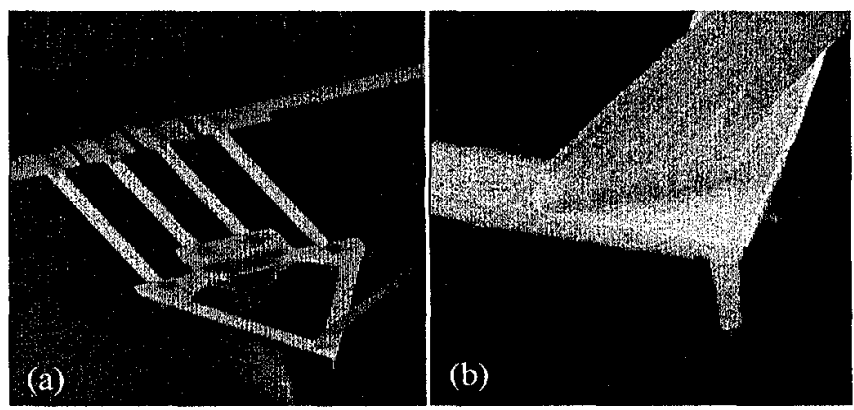

Figure 4. (a) SEM image of the force sensor with a seta. (b) Closeup at the sensor tip shows detailed structure of the spatulae. 
The entire device is made of single crystalline silicon. It has two independent force sensors, each with one predominant direction of compliance. The vertical sensor consists of a thin triangular probe, and the lateral sensor consists of four tall slender ribs. A special $45^{\circ}$ oblique ion implantation allows piezoresistive and conductive regions to be implanted on both horizontal and vertical surfaces simultaneously. Forces applied to the tip of the sensor are resolved into these two orthogonal directions, and can be measured by the changes in resistance of the piezoresistors. Since this device is originally designed for data storage applications, each cantilever contains a sharp conical tip at the apex of its triangular probe. Therefore the tipless backside of this device is used to provide a flat surface for setal adhesion. The backside of the sensor has a surface RMS roughness of less than $2.5 \mathrm{~nm}, 2$ orders of magnitude smaller than the size of the spatula tips on setae. Therefore it is safe to assume that micro-interlocking does not play a major role in the adhesion.

Typical noise spectra for this device are shown in Figure 5. The dotted line in each graph corresponds to the Johnson noise floor for a resistor value equal to the nominal resistance of the piezoresistor. The integrated noise level in each piezoresistor is equal to a minimum detectable force of $\sim 5 \mathrm{nN}$ in a $10 \mathrm{kHz}$ bandwidth, more than sufficient for measuring the $\mu \mathrm{N}$-scale adhesion of individual setae.

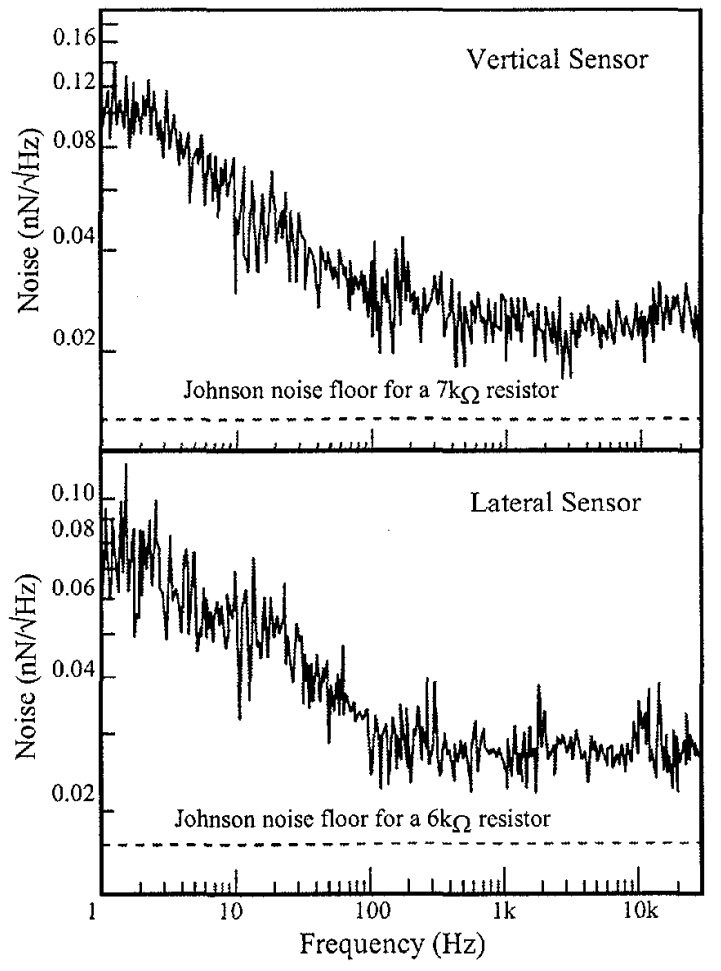

Figure 5. Noise spectra of the dual-axis force sensor.

\section{EXPERIMENTAL SETUP}

A single seta, peeled off of the toe of a $\sim 100-g$ Tokay gecko, is carefully glued to the tip of a $\# 2$ insect pin using 5 -minute epoxy. The pin is mounted on a computer-controlled piezoelectric manipulator (Melles Griot, NanoFlex TM) and orientated so that the active surface of the seta is facing down. The sensor is mounted on a movable microscope stage with the desired surface facing up. The seta is brought into contact with the sensor from above and a small downward preload is applied to initiate adhesion. The seta is then pulled away.

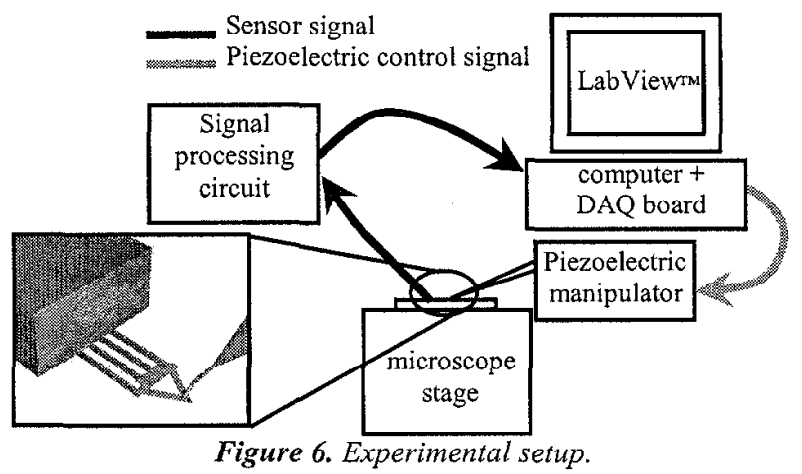

Sensor signals in both directions are amplified and filtered through a pair of $300-\mathrm{Hz}$ low-pass filters, and digitized at $100 \mathrm{~Hz}$ using LabView TM on a PC with a 16-bit data acquisition board (National Instrument PCI-MIO-16XE-50). The collected data are then converted to sensor deflections through calibration constants, and multiplied by cantilever stiffness to obtain force data.

\section{RESULTS}

Figure 7 shows the top view of the first configuration used to study the dynamics of this adhesion-breakaway event. The seta is brought into contact with the tip area of the sensor for simultaneous vertical and lateral force measurements.

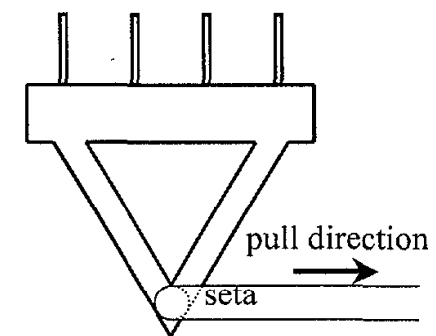

Figure 7. Experimental configuration $\# 1$.

After a small vertical preload is applied, the seta is pulled away laterally at a rate of $5 \mu \mathrm{m} / \mathrm{sec}$. This pulling action is equivalent to a linearly increasing applied lateral force. Dellit [9] suggested that tension on the setae in this direction is necessary to initiate traction.

Once the pulling begins, the lateral sensor deflects with the pull until the pulling force overcomes adhesion. At this point the seta loses adhesion and breaks away from the sensor. The cantilever springs back to its initial position and the sensor signal returns to zero. The force measured at the point of breakaway is the adhesion capacity of the seta on this surface. A typical force curve in this configuration is shown in Figure 8 . The slight buckling of the seta due to the preload pushes the sensor to the left and causes an initial negative lateral force of $\sim 30 \mu \mathrm{N}$. In this figure, a maximum lateral force of $\sim 45 \mu \mathrm{N}$ (point $\mathrm{C}$ ) is achieved with a $\sim 5 \mu \mathrm{N}$ preload.

Figure 8 also shows discrete sections of varying slopes on the lateral force curve. From these different sections, we can hypothesize the dynamics of the adhesion-breakaway events as 
follows: Point A denotes the start of the pulling motion. As the pulling continues, lateral force builds up linearly, as evident from the somewhat constant positive slope between $\mathrm{A}$ and $\mathrm{B}$. At $\mathrm{B}$, there is a significant drop in the slope of the lateral force curve, indicating slippage between the seta and sensor. Since the active surface of the seta is comparable in size to the surface area at the sensor tip, the seta begins to slide off the edge of the sensor almost as soon as it begins to slip, therefore reducing the contact area. However, despite the reduction in contact area, lateral force continues to build up as pulling continues between B and $C$. This suggests that the adhesive force per area must be increasing, since the total adhesive force is the product of force per area and the contact area. At point $\mathrm{C}$, most of the seta surface has lost contact with the sensor. The remaining part can no longer support the sensor deflection. At this point the seta breaks loose from the sensor. The sensor then recoils, occasionally hindered by parts of the seta still touching its top surface, before returning to its unloaded position at $\mathrm{D}$.

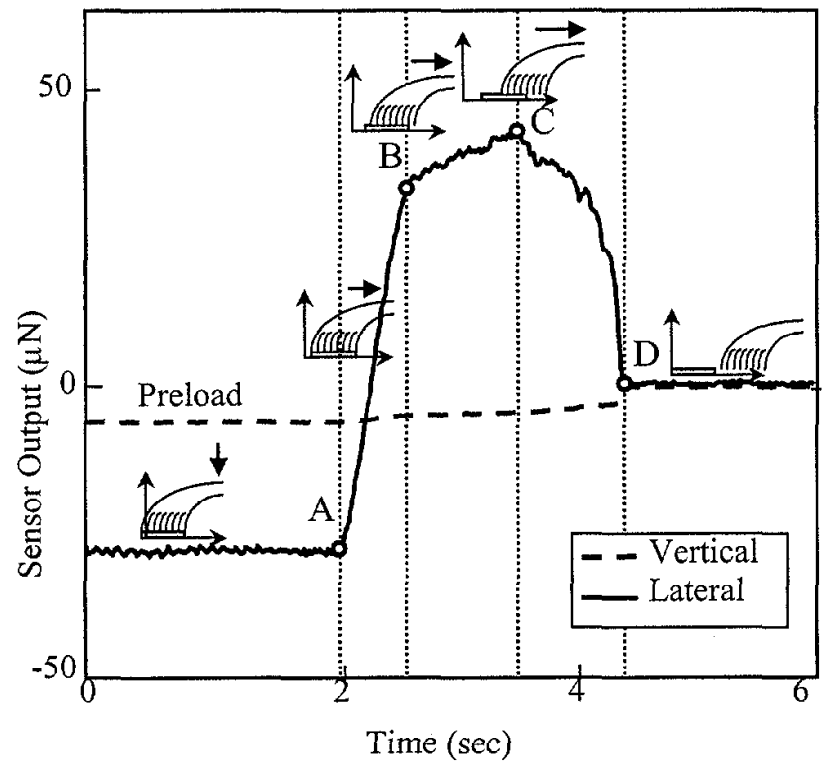

Figure 8. Force curve obtained using configuration \#1 and schematics showing detachment events.

If this hypothesis were true, we have just revealed an interesting possible mechanism by which geckos possess such remarkable wall-climbing ability: a fraction of the spatulae at the setal tip will adhere to the surface at initial contact. If the initial adhesion is not sufficient to sustain a gecko's body weight, the animal begins to slide down the wall, cquivalent to the sliding of a single seta on the sensor surface in our experiment. It is possible that initially attached spatulae will force their unattached neighboring spatulae to become engaged in the adhesion through sliding. Thus more and more spatulae will adhere to the surface as the pulling motion continues, building up adhesion, until the force is great enough to support the animal's weight. Note that the sliding distance necessary for building up to maximum adhesion is very small, on the order of a few microns.

A second set of experiments was performed using configuration \#2, shown in Figure 9, to support this hypothesis. In order to provide a larger surface for sliding, the base of the triangular probe region on the sensor is used. In this configuration the vertical sensor is not accessible, but the setae are able to slide on the sensor surface and build up much larger adhesion forces.

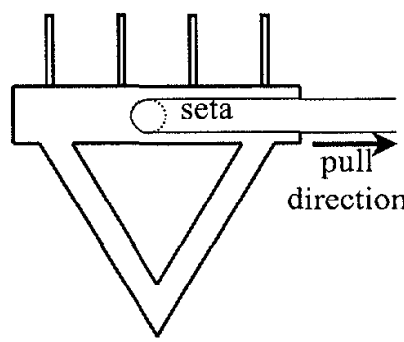

Figure 9. Experimental configuration \#2.

A typical force curve obtained in this configuration, shown in Figure 10, shows a maximum adhesion force of nearly $180 \mu \mathrm{N}$.

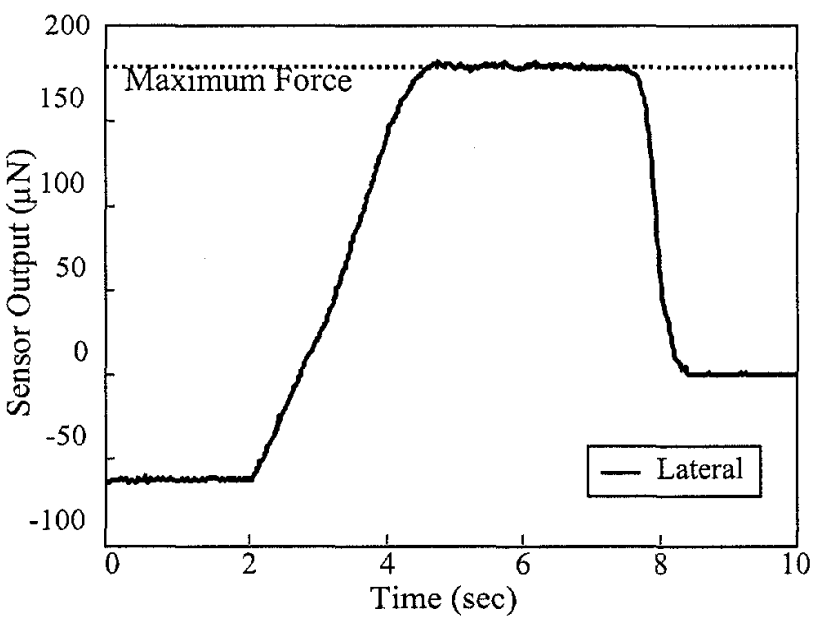

Figure 10. Force curve obtained using configuration \#2.

Note that in Figure 10, a constant maximum force is maintained until detachment, when the seta slides off the edge of the sensor. The maximum force presumably is reached when nearly all of the spatulae are in contact with the sensor surface. Even though the vertical preload is unknown, the maximum force in Figure 10 is several times larger than can be obtained when the seta is placed at the tip of the sensor with any preload. This demonstrates that setal adhesion does build up significantly through sliding.

\section{Preload}

To study the effect of preload, the detachment force, or the adhesive forces at first slippage (point $B$ in Figure 8), are recorded and plotted against various preload values in Figure 11. A clear positive correlation between adhesion and preload can be seen. This experiment shows that preloading improves the initial contact between the seta and the surface. To rule out friction as the dominating adhesion mechanism, we also measured the friction coefficient between the inactive non-spatular backside of the seta and the same surface on the sensor. The coefficient of friction between this silicon/keratin interface is measured to be approximately $\approx 0.2$. The magnitude of frictional force is drawn as the dashed line near the bottom of the graph, and clearly indicates that friction plays a minimal role in setal adhesion.

Preload of above $15 \mu \mathrm{N}$ causes the seta to buckle. This introduces an unnecessary complexity in the experiment. Therefore only data with preload values less than $15 \mu \mathrm{N}$ are 
plotted. Figure 11 consists of 42 data points taken using the same seta in one sitting, therefore minimizing variations in all other parameters, and demonstrating an apparent positive relationship between preload and adhesive forces. The number of data points that can be reliably taken using one seta is limited because setae tend to dry out and lose adhesion when exposed to the strong light on the microscope for an extended period of time.

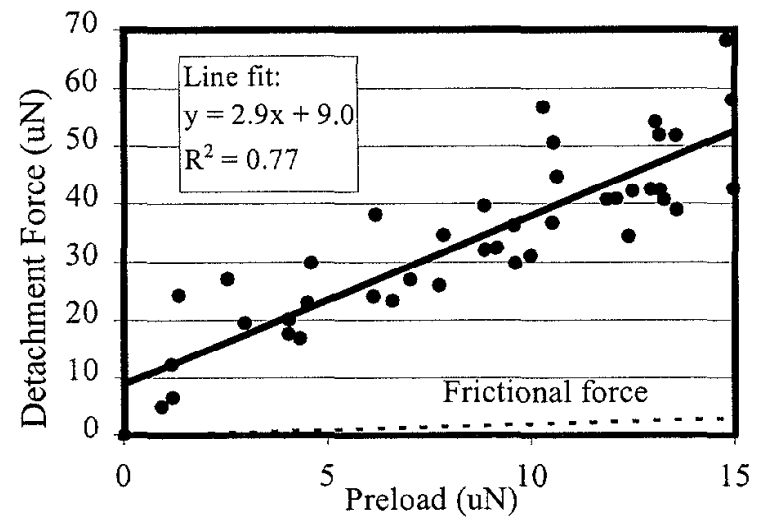

Figure 11. Detachment force as a function of preloads. $(N=42)$

This dependency is presumably due to the larger number of spatulae in initial contact with the surface with larger preload. The effect of preloading on setal adhesion supports the hypothesis that a small perpendicular force in concert with a rearward displacement may be neccssary to engage adhesion [4]. Experiments were repeated on several other setae which all showed a positive correlation between preload and adhesion.

\section{Pulling Speed}

We also performed a series of experiments using the second configuration (Figure 9) to study the effect of pulling speed on the force buildup through sliding. The magnitude of the preload is unknown, but kept constant throughout the experiment.

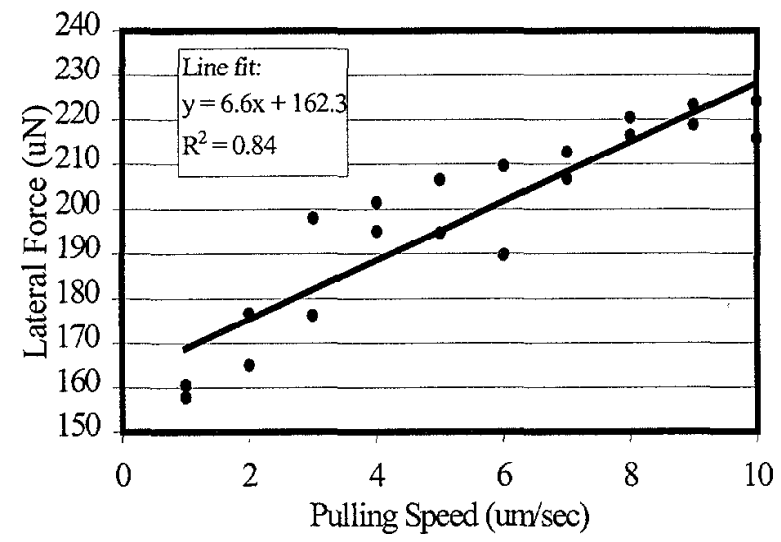

Figure 12. Maximum lateral force with sliding build-up. $(N=18)$

Figure 12 shews the maximum adhesive forces (dotted line in Figure 10) obtained at pulling speeds varying from 1 to 10 $\mu \mathrm{m} / \mathrm{sec}$. Each point represents a single data point. All 18 points are taken using the same seta in one sitting. Maximum pulling speed is limited by the response time of the piezoelectric manipulator used to pull the setae. Again a clcar positive correlation can be scen. As discussed previously in the results section, initially unattached spatulae can be pulled into contact with the surface by their neighbors through sliding. It is possible that somehow a higher pulling rate increases this dynamic effect. More experiments are necessary to support this hypothesis.

\section{DISCUSSION}

The magnitude of the lateral adhesive force measured for each seta depends greatly on the size and orientation of its active surface. In general larger forces are measured when a larger area of the setal active surface is in contact with the sensor surface. Experimental data showed that adhesion increases with increasing preload and pull speed. Most importantly, adhesion increases significantly if the seta is dragged a short distance across the surface. All these can be attributed to the increase in number of spatulae that come in contact with the surface, which supports the hypothesis that the wall-climbing ability of geckos is likely due to van der Waals forces. Furthermore, the measured contact forces are consistent with the predicted levels of van der Waals forces between these two surfaces.

Our data also showed that geckos are overbuilt in their adhesive capacity. If all setae on the gecko can provide up to $\sim 200$ $\mu \mathrm{N}$ of force, an entire animal can sustain $200 \mathrm{~N}$ of force with one million setae, orders of magnitude larger than the animal's total body weight. The elaborate pad structure with microscopic setae is responsible for this remarkable clinging ability. We have also found that setae can still stick to surfaces very well more than one month after they have been peeled off of the animals, as long as they are kept moist for the duration.

\section{CONCLUSION}

All of our experimental results support the van der Waals assumption, but further experiments are necessary to confirm this theory. We are in the process of fabricating an improved version of cantilevers designed specifically for this application. These new cantilevers have been redesigned and optimized for the desired force range [17], and they will also have wider tip areas for studying the effect of sliding while simultaneously measuring vertical and lateral forces. Possible future directions also include measuring vertical detachment forces, correlating the pulling angle to the maximum adhesion, as well as varying surface material and roughness by depositing various coatings on the sensor surface.

We have demonstrated the use of a novel dual-axis MEMS cantilever for high-quality measurement of dry adhesion on gecko setae. Several interesting characteristics of the setae are revealed based on data obtained using this MEMS device. In addition to addressing an important and long-standing controversy in the animal biomechanics community, this work illustrates an important application area for MEMS devices - the measurement of relatively small forces in biomechanical structures. Experiments using the newly fabricated cantilevers will allow us to perform more extensive study on the dynamics of adhesion. We also seek further opportunities for MEMS measurement capabilities to address biomechanical measurement problems.

\section{ACKNOWLEDGMENTS}

The authors would like to thank Ben Chui, Eugene Chow, and Molly McGuire for their assistance on this project. This project is funded in part by the NSF XYZ On-a-Chip Program, the Office of Naval Research MURI for Biomimetic Robotics (N00014-98-1-0669), the National Science Foundation Graduate 
Research Fellowship, and the National Science Foundation CAREER Award (ECS-9502046). Fabrication of the MEMS device made use of the Stanford Nanofabrication Facility that is a part of the National Nanofabrication Users Network funded by the National Science Foundation under award number ECS-9731294.

\section{REFERENCE}

1. K. Autumn, et al., "Adhesive Force of a Single Gecko FootHair", accepted by Nature for publication (2000).

2. B. W. Chui, et al., "Independent detection of vertical and lateral forces with a sidewall-implanted dual-axis piezoresistive cantilever", Applied Physics Letters, 72(11): 1388-1390 (1998).

3. D. J. Irschick, et al., "A comparative analysis of clinging ability among pad-bearing lizards", Biological Journal of the Linnean Society, 59(1): 21-35 (1996).

4. R. Ruibal and V. Ernst, "The Structure of Digital Setae of Lizards", Journal of Morphology, 117: 271-294 (1965).

5. A. P. Russel, "A Contribution to the Functional Analysis of the Foot of the Tokay, Gekko-Gecko (Reptilia, Gekkonidae)", Journal of Zoology London, 176(4): 437-476 (1975).

6. B. C. Madhendra, "Contribution to the Bionomics, Anatomy, Reproduction, and Development of the Indian House-Gecko Hemidactylus flaviviridis Ruppell. Part II. The problem of locomotion", Proc. Indian Acad. Sci., 3: 288-306 (1941).

7. H. Gadow, The Camhridge Natural History Vol. 8: Amphibia and Reptiles, London, McMillan and Co. (1901).

8. J. F. Gennaro, "The Gecko Grip", Natural History, 78: 36-43 (1969).

9. W. F. Dellit, "Zur Anatomie und Physiologie der Geckozehe", Jena. Z. Naturw, 68: 613-656 (1934).

10. A. Bellairs, The Life of Reptiles, New York, Universe Books (1970).

11. P. F. A. Maderson, "Keratinized Epidermal Derivatives as an Aid to Climbing in Gekkonid Lizards", Nature, 203: 780-781 (1964).

12. H. R. Schmidt, "Zur Anatomie und Physiologie der Geckopfote", Jena Z. Naturw, 39: 551 (1904).

13. U. Hiller, "Untersuchung zum Feinbau und zur Funktion der Haftborsten von Reptilien", Zeitschrift fur Morphologie der Tiere, 62(307-362): (1969).

14. J. T. Feddema, et al., "Micro-Assembly Planning with van der Waals Force", Proceedings of the IEEE International Symposium on Assembly and Task Planning, Porto, Portugal (1999), pp. 3238.

15. J. N. Israelachvili, Intermolecular And Surface Forces, 2nd Edition, London, Academic Press (1992).

16. R. E. Baier, et al., "Adhesion: Mechanisms That Assist or Impede It", Science, 162: 1360-1368 (1968).

17. Y. Liang and T. Kenny, "Mechanical Optimization of a DualAxis Piezoresistive Force Sensor", Proceedings of the ASME International Congress and Exposition MEMS Symposium, Anaheim (1998). 